\title{
Unusual Presentation of Traumatic Neuroma on the Gingiva Following a Road Traffic Accident - A Case Report
}

\author{
Hamad Alzoman ${ }^{1}$, Khalid Binsaeed ${ }^{2}$, Rana Alshagroud ${ }^{3}$, Ra'ed Alsadhan ${ }^{4}$, Tahsinul Haque ${ }^{5}$ \\ ${ }^{1}$ Department of Periodontics and Community Dentistry, College of Dentistry, King Saud \\ University, Riyadh, Kingdom of Saudi Arabia. ${ }^{2}$ College of Dentistry, King Saud University, \\ Riyadh, Kingdom of Saudi Arabia. 3,4,5 Department of Oral Medicine and Diagnostic \\ Sciences, College of Dentistry, King Saud University, Riyadh Kingdom of Saudi Arabia.
}

\section{INTRODUCTION}

Traumatic neuroma is a tumour-like swelling, characterized by localized reactive proliferation of the peripheral nerve occurring in response to transection or other damage to the nerve bundles. It represents an ineffective attempt at nerve repair following trauma that results in degeneration of the nerve followed by nerve axon regeneration and remyelination within the dense fibrous connective tissue stroma. ${ }^{1}$ The most common cause of nerve injury is surgical treatment; however, other causes have been reported such as lack of blood supply, pressure, cuts, tissue injuries, and bleeding into the neighbouring area. ${ }^{2}$ In addition, other aetiological factors may result in the formation of a TN if occurred in proximity to the sensory nerve such as: chronic irritation, abnormal wound healing, injection of dental anaesthesia, or atypical scar formation. ${ }^{3}$

TN rarely occurs in the oral cavity; ${ }^{4}$ however, it has been noted in the area of the mental foramen, lower lip and tongue.5,6 Only few cases of TN have been described on the palate. ${ }^{7}$ Tamiolakis et al have reported the prevalence of oral neural tumours to be $0.4 \%$ out of 35,590 collected biopsies. Only 25 cases were diagnosed as traumatic neuroma out of the total number of biopsies. None of the reported TN cases were from the gingiva region. ${ }^{8}$ To our knowledge, there have been no previous reports of a TN in the region of the gingiva with an atypical clinical appearance.

In this report, we describe a case of $\mathrm{TN}$ of the mental nerve with an unusual clinical appearance in the region of the gingiva in a patient with a history of a road traffic accident.

\section{PRESENTATION OF CASE}

A 46-year-old male, non-smoker, presented to the Dental University Hospital, King Saud University, Riyadh, for a routine dental care. He reported a negative medical history and was not taking any medications. During intraoral examination, a gingival swelling was observed between the mandibular right canine and the first premolar. The lesion was a $12 \mathrm{~mm} \times 5 \mathrm{~mm}$, pink, firm, smooth soft tissue swelling covered by a normal mucosa circumferentially (Fig. 1). The lesion had a peculiar appearance; it was attached to the gingival tissue in two positions; coronally, attached to the dental papilla between the canine and premolar and apically, attached to the alveolar mucosa. The intermediate part was free from the underlying tissues forming a tunnellike area through which a probe was passed freely (Fig. 2). To our knowledge, this unusual clinical presentation has not been hitherto reported.

Upon further review of the medical history, the patient mentioned that he had been in a motor vehicle accident 27 years ago. He sat at the backseat and suffered facial injuries as a result of the accident. He was hospitalized for seven days for the treatment of a dentoalveolar fracture of the mandible due to the accident. In addition, the accident caused a fracture of the maxillary right central incisor, an avulsion of the mandibular right lateral incisor, and an obvious deviation of the mandibular incisors.

After a considerable period, he noticed an alteration of sensation in the right side of his lower lip, but he did not seek treatment since he got used to the lower lip paraesthesia.

\author{
Corresponding Author: \\ Dr. Hamad Alzoman, \\ BDS, M.S., Associate Professor, \\ Diplomate American Board of \\ Periodontology, Department of \\ Periodontics and Community \\ Dentistry, College of Dentistry, \\ King Saud University Riyadh, \\ Saudi Arabia. \\ E-mail: halzoman@ksu.edu.sa
}

DOI: $10.14260 /$ jemds/2021/86

How to Cite This Article:

Alzoman H, Binsaeed K, Alshagroud R, et al. Unusual presentation of traumatic neuroma on the gingiva following a road traffic accident - a case report. J Evolution Med Dent Sci 2021;10(06):391-393, DOI: 10.14260/jemds/2021/86

Submission 06-10-2020,

Peer Review 09-12-2020,

Acceptance 15-12-2020,

Published 08-02-2021.

Copyright (c) 2021 Hamad Alzoman et al. This is an open access article distributed under Creative Commons Attribution License [Attribution 4.0 International (CC BY 4.0)] 

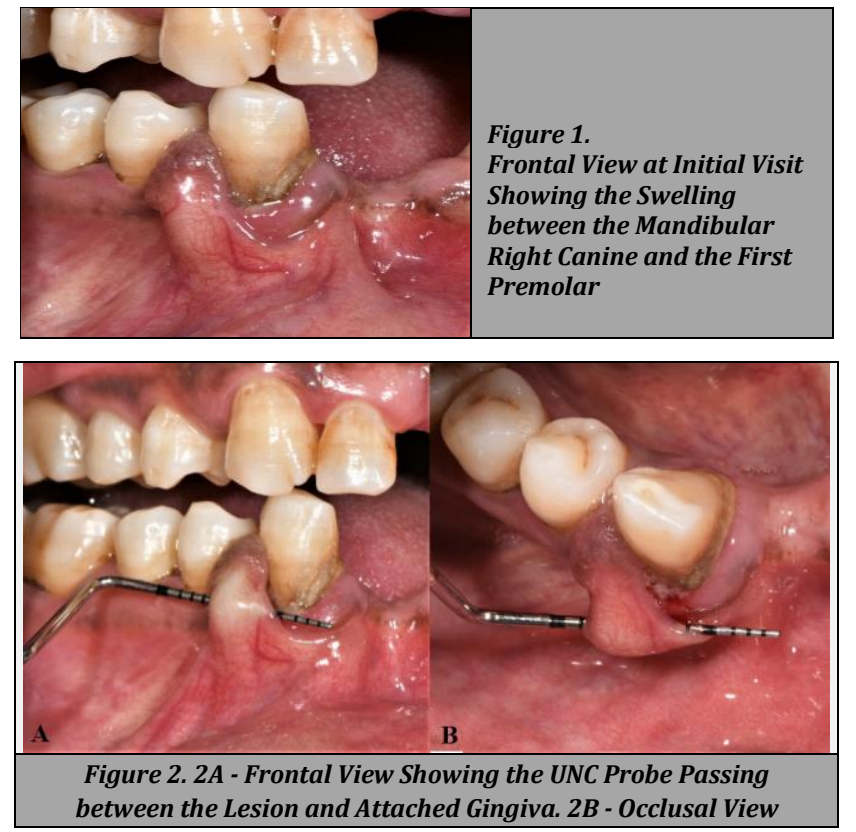

\section{Radiographic Examination}

The radiographic assessment consisted of panoramic, periapical, bitewing and Cone Beam Computed Tomography (CBCT). The images showed intact buccal cortical plate and no effect of the lesion on the alveolar ridge (Fig. 3).

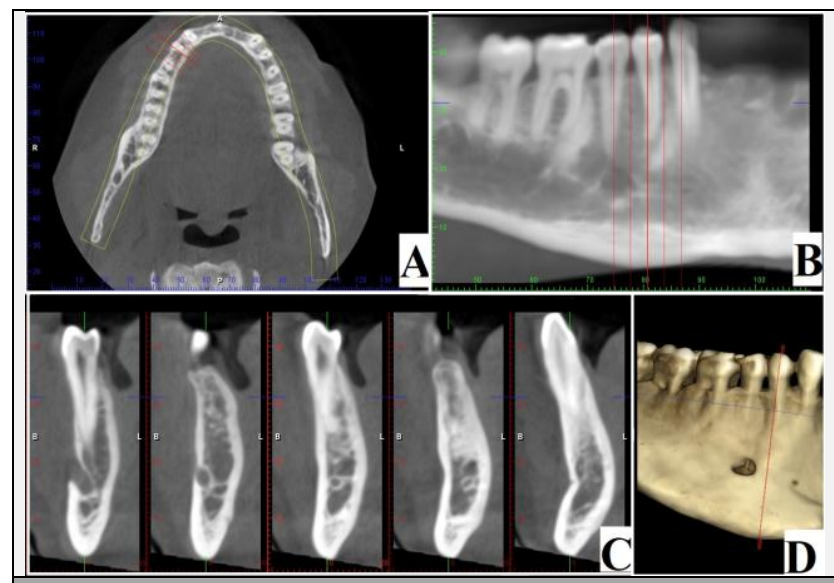

Figure 3. CBCT Examination of the Right Mandibular Body in Axial Section (A) as well as in Curved Multiplanar Reconstruction Panoramic (B) and Buccolingual Cross Sections (C). Levels of the Buccolingual Sections are Shown as Red Lines on the Sections and on the Volume Rendered Reconstruction of the Mandible (D). Image Showing Intact Cortical Bone and No Effect of the Lesion on the Alveolar Ridge of the Mandible.

\section{DISCUSSION OF MANAGEMENT}

After a case presentation to the patient and consenting, the patient underwent excisional biopsy under local anaesthesia with $2 \%$ lidocaine with 1:200,000 epinephrine. A vertical incision was performed at the long axis of the lesion followed by dissection of the lesion using blade \#15. The lesion was separated from the surrounding connection tissue and completely excised. It was sent in $2 \%$ formalin container to the histopathology laboratory for investigation. Several simple interrupted sutures were placed using 5 - 0 proline (Fig. 4). To relieve post-operative pain, Ibuprofen (Brufen, Reckitt
Benckiser Healthcare International) $600 \mathrm{mg}$ was prescribed three times daily for five days and post-operative instructions were given to the patient. The histopathology report described a haphazard proliferation of mature myelinated and unmyelinated nerve bundles within a fibrous connective tissue stroma. Some of the nerve bundles were surrounded by prominent perineurium suggesting TN. Immunohistochemistry evidenced strong S-100 and CD57 positivity of the cells within the lesion. EMA (Epithelial Membrane Antigen) used to highlight the perineurium around the nerve bundles (Fig. 5).

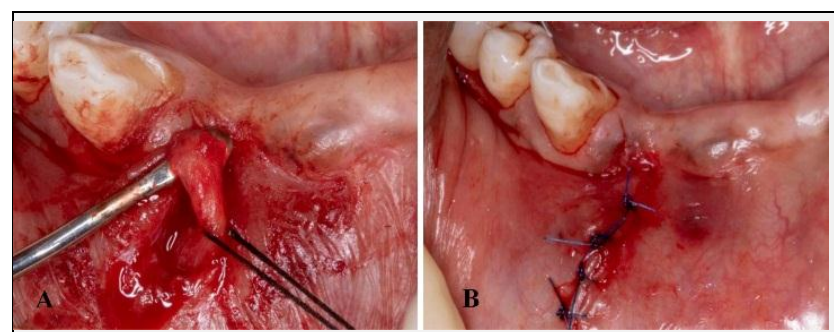

Figure 4. 4A - The Lesion was Retracted after Separating from Surrounding Connective Tissue. 4B -After Excision, Simple Interrupted Suture was Performed Using 5 - 0 Proline

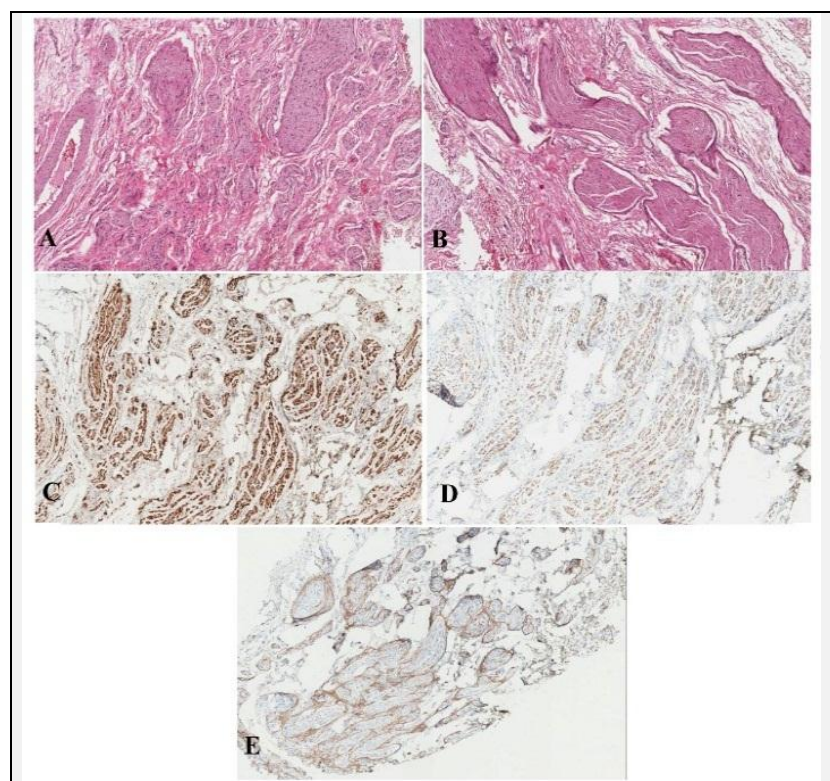

Figure 5. 5A - Histopathological Examination Showing a Haphazard Arrangement of Nerve Bundles within a Fibrous Connective Tissue Stroma. Hematoxylin and Eosin Stain. Magnification $\times 200.5 B-$ Nerve Bundles Surrounded by Perineurium. Hematoxylin and Eosin Stain Magnification $\times 200$. 5C -Strong Positivity of the Bundles for the Neural marker S-100. Immunohistochemical Staining. Magnification $\times 200.5 D$ - Positivity of the Bundles for the Neural marker CD57. Immunohistochemical Staining. Magnification $\times 200$. 5E - EMA was Positive in the Perineurium. Immunohistochemical Staining. Magnification $\times 200$

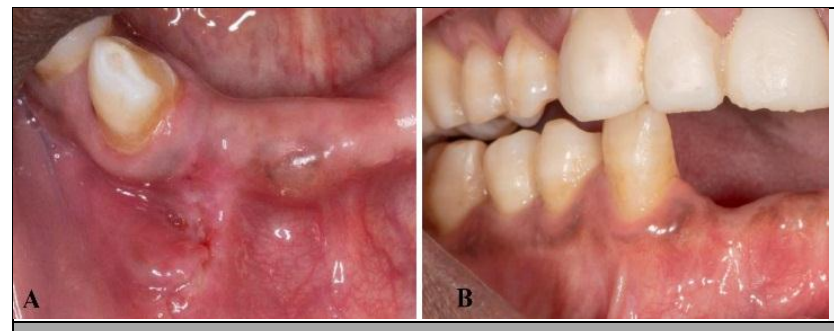

Figure 6, 6A - At 1 Week Follow-Up - Uneventful Healing of the Surgica Site after Removal of Suture. $6 B$-At 4 Months Follow-Up - Complete Recovery without Recurrence of the Lesion. 


\section{Clinical Outcomes}

At one week of follow-up, it was observed that the surgical site was properly healed and the sutures were removed (Fig. 6A). At four-month follow-up, it was observed that there was no recurrence of lesion, no alteration of sensation, and the patient was completely asymptomatic (Fig. 6B).

$\mathrm{TN}$ is a reactive proliferation of neural tissue after nerve bundle interruption or damage that have been reported to occur following tooth extraction, sagittal split osteotomy and chin augmentation. ${ }^{9,10}$ In the present case, the lesion was not associated with any of the previously mentioned factors. The patient however, had a history of a road traffic accident and this was assumed to be the cause of trauma. TN can occur at any age. However, they are most often diagnosed in young and middle-aged adults. 6,8 Clinical findings of TN may differ. Soft tissue TN may present smooth-surfaced, non-ulcerated, palpable and slow growing nodules. Pain, tenderness and paresthesia are the most common signs and symptoms of TN and, it could be asymptomatic in $25 \%$ cases. ${ }^{11-13}$ In the present case, the patient experienced altered sensation of the lower lip. There are very few studies explaining the mechanism of altered sensation or paraesthesia in TN. Nordin et al. reported that lowering the threshold of large myelinated nerves that produce ectopic impulses may lead to sensation alteration. ${ }^{14}$ It has also been reported that neuroglial cells specially satellite cells in the sensory ganglia play an important role in neuropathic conditions. ${ }^{15}$

In this case, it is assumed that $\mathrm{TN}$ occurred due to a road traffic accident that subsequently resulted in tissue damage in the area of the mental nerve and the surrounding gingival tissue. We observed that the discomfort and abnormal sensation in the mental nerve region were eliminated completely after surgical excision of the lesion. This is a clinical observation that has not been previously reported.

Financial or other competing interests: None.

Disclosure forms provided by the authors are available with the full text of this article at jemds.com.

\section{REFERENCES}

[1] Zachariades N, Mezitis M, Vairaktaris E, et al. Benign neurogenic tumours of the oral cavity. Int J Oral Maxillofac Surg 1987;16(1):70-6.
[2] Jham BC, Costa NL, Batista AC, et al. Traumatic neuroma of the mandible: a case report with spontaneous remission. J Clin Exp Dent 2014;6(3):e317-20.

[3] Foltan R, Klima K, Spackova J, et al. Mechanism of traumatic neuroma development. Med Hypotheses 2008;71(4):572-6.

[4] Alotaiby FM, Fitzpatrick S, Upadhyaya J, et al. Demographic, clinical and histopathological features of oral neural neoplasms: a retrospective study. Head Neck Pathol 2019;13(2):208-14.

[5] Tokuc B, Altindis S, Coskunses FM, et al. Excision of rare intraosseous traumatic neuroma of the mandible. J Stomatol Oral Maxillofac Surg 2020;S24687855(20)30206-8.

[6] Alotaibi O, Al Sheddi M. Neurogenic tumours and tumour - like lesions of the oral and maxillofacial region: a clinicopathological study. Saudi Dent J 2016;28(2):76-9.

[7] Eguchi $\mathrm{T}$, Ishida $\mathrm{R}$, Ara $\mathrm{H}$, et al. A diffuse traumatic neuroma in the palate: a case report. J Med Case Rep 2016;10(1):116.

[8] Tamiolakis P, Chrysomali E, Andrikopoulou AS, et al. Oral neural tumours: clinicopathologic analysis of 157 cases and review of the literature. J Clin Exp Dent 2019;11(8):e721-31.

[9] Arribas-Garcia I, Alcala-Galiano A, Gutierrez R, et al. Traumatic neuroma of the inferior alveolar nerve: a case report. Med Oral Patol Oral Cir Bucal 2008;13(3):E186-8.

[10] Sayan NB, Ucok C. Asymptomatic traumatic neuroma after mandibular sagittal split osteotomy: a case report. J Oral Maxillofac Surg 2002;60(1):111-2.

[11] Deng W, Chen SL, Huang DY. Traumatic neuroma of mental nerve following chin augmentation. Int J Oral Maxillofac Surg 2009;38(12):1324-6.

[12] Yao C, Zhou X, Zhao B, et al. Treatments of traumatic neuropathic pain: a systematic review. Oncotarget 2017;8(34):57670-9.

[13] Kodama Y, Seo K, Hayashi T, et al. Orofacial pain related to traumatic neuroma in a patient with multiple TMJ operations. Cranio 2012;30(3):183-7.

[14] Nordin M, Nystrom B, Wallin U, et al. Ectopic sensory discharges and paresthesiae in patients with disorders of peripheral nerves, dorsal roots and dorsal columns. Pain 1984;20(3):231-45.

[15] Chiang CY, Dostrovsky JO, Iwata K, et al. Role of glia in orofacial pain. Neuroscientist 2011;17(3):303-20. 